\title{
Lattice Coulomb propagators, effective energy and confinement
}

\author{
Giuseppe Burgio* \\ Institut für Theoretische Physik \\ Auf der Morgenstelle 14 \\ 72076 Tübingen \\ Germany \\ E-mail: giuseppe.burgio@uni-tuebingen. de
}

\section{Markus Quandt}

Institut für Theoretische Physik

Auf der Morgenstelle 14

72076 Tübingen

Germany

E-mail: markus.quandt@uni-tuebingen.de

\section{Hugo Reinhardt}

Institut für Theoretische Physik

Auf der Morgenstelle 14

72076 Tübingen

Germany

E-mail: hugo.reinhardteuni-tuebingen. de

\section{Mario Schröck}

Institut für Physik, FB Theoretische Physik

Universität Graz

8010 Graz

Austria

E-mail: mario.schroeck@uni-graz.at

We show that in the lattice Hamiltonian limit all Coulomb gauge propagators are consistent with the Gribov-Zwanziger confinement mechanism, with an IR enhanced effective energy for quarks and gluons and a diverging ghost form factor compatible with a dual-superconducting vacuum. Multiplicative renormalizability is ensured for all static correlators, while for non-static ones their energy dependence plays a crucial role in this respect. Moreover, from the Coulomb potential we can extract the Coulomb string tension $\sigma_{C} \sim 2 \sigma$.

Xth Quark Confinement and the Hadron Spectrum

8-12 October 2012

TUM Campus Garching, Munich, Germany

\footnotetext{
* Speaker.
} 


\section{Introduction}

QCD in Coulomb gauge, being best suited to examine the Gribov-Zwanziger (GZ) confinement ideas [1,2], has been the subject of intense research in the last few years. In a series of papers $[3,4,5,6,7,8]$, which we will briefly summarize here, we have analyzed the behaviour of all relevant two-point functions on the lattice and compared them with the corresponding predictions of Hamiltonian variational calculations $[9,10,11]$, concentrating on the features relevant for the GZ scenario.

As Gribov in his seminal paper noticed, for non-Abelian theories most gauge conditions admit several solutions and the corresponding Faddeev-Popov (FP) mechanism is not sufficient to define the functional integral beyond perturbation theory. The field-configuration space must therefore be restricted to a domain, continuously connected to the origin, where the gauge condition at hand always possesses unique solutions. He then argued how, as soon as the fields cross the boundary of such region, the ghost dressing function acquires a singularity; the "no-pole" condition for the FP-ghost is then necessary to implement the restriction. In particular, in Coulomb gauge, he argued how such restriction can imply a diverging gluon self-energy, motivating its disappearance from the physical spectrum.

Many issues remain of course in the above description open. Gribov based his conjectures on more or less heuristic arguments. Zwanziger later tried to put the whole set-up on a more solid basis, while variational calculations, which are viable in Coulomb gauge since they by-pass the explicit construction of the gauge invariant Hilbert space [12], did provide some insight on the relation of the GZ-mechanism to the Hamiltonian formulation. In both cases, however, approximations need to be made; although many authors tackled the problems during the years $[13,14,15,16,17,18]$, a satisfactory non-perturbative cross-check from lattice calculation was hindered for a long time by the presence of strong discretization effects. In our papers we have shown $[3,7,8]$ how for each propagator improvement techniques can quite effectively take care of such problems and make an explicit check of the GZ-scenario possible.

As first suggested in [3], the size of discretization effects can be investigated on anisotropic lattices, where the time and space like cut-off $a_{t}, a_{s}$ are kept different. In Fig. 1 we show the effect of taking the limit $a_{t} \rightarrow 0$, which controls the lattice Hamiltonian limit, on the SU(2) Coulomb gauge functional calculated at fixed space-like cut-off, i.e. RG-point. Perturbative determinations of the latter $[19,20]$ are not precise enough and we have re-checked the only non-perturbative calculation found in the literature [21]; details can be found in [8], as well as the details of the gauge fixing algorithm, which adapts those introduced in [22, 23]. Following the ideas in [3], a first anisotropic analysis in SU(3) had been attempted in [24].

From the continuum analysis and from our results in $[3,4]$ we know that in the pure gauge sector the static gluon propagator, the static Coulomb potential and the ghost form factor should obey:

$$
\begin{aligned}
& D(\vec{p})=\frac{|\vec{p}|}{\sqrt{|\vec{p}|^{4}+M^{4}}} \\
& V_{C}(\vec{p})=\frac{8 \pi \sigma_{C}}{|\vec{p}|^{4}}+\frac{\eta}{|\vec{p}|^{2}}+\mathscr{O}(1) \\
& d(\vec{p}) \simeq\left\{\begin{array}{cl}
\frac{1}{|\vec{p}|^{\mathrm{K}_{\mathrm{gh}}}} & |\vec{p}| \ll \Lambda \\
\frac{1}{\log _{\mathrm{gh}} \frac{|\vec{p}|}{m}} & |\vec{p}| \gg \Lambda
\end{array}\right.
\end{aligned}
$$




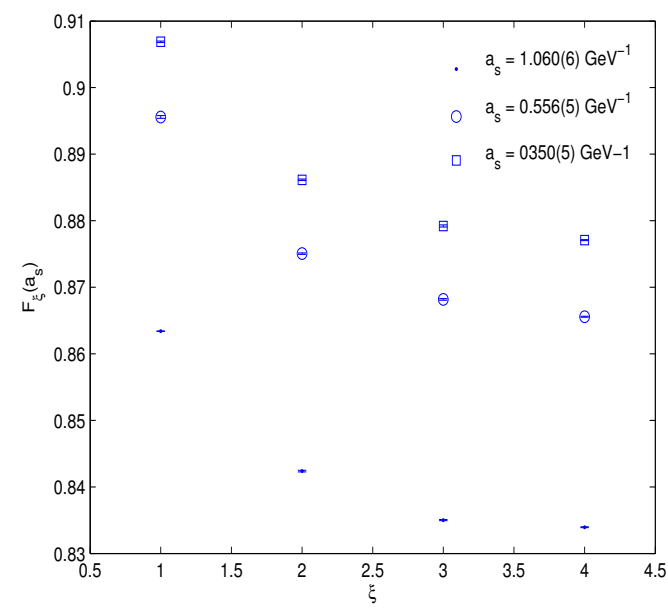

(a)

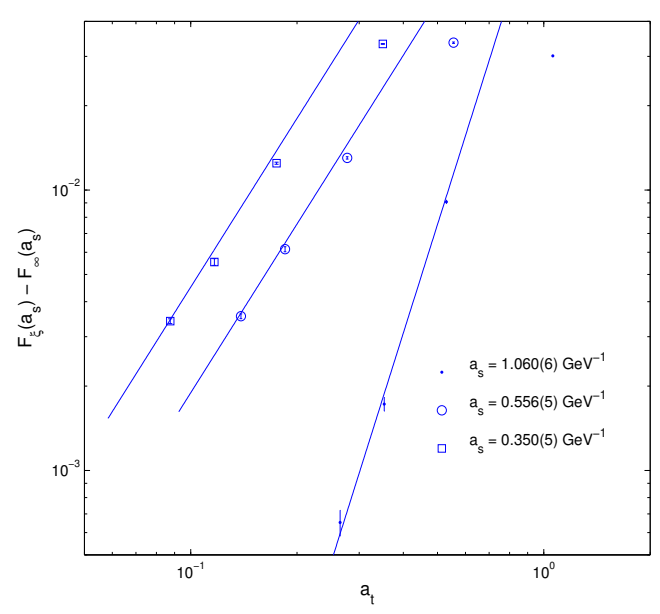

(b)

Figure 1: (a): dependence of the gauge fixing functional $F_{\xi}\left(a_{s}\right)$ on the anisotropy $\xi=a_{s} / a_{t}$ at fixed spatial cut-off $a_{s}$. (b): Deviation of the gauge fixing functional from the Hamiltonian limit, $F_{\xi}\left(a_{s}\right)-F_{\infty}\left(a_{s}\right)$, as a function of the temporal lattice spacing $a_{t}$, together with its leading power corrections.

where $M \simeq 1 \mathrm{GeV}$ and for the gluon self-energy $\omega_{A}=D^{-1}(\vec{p})$. The quark propagator, the fermion self energy and the running mass $M(|\vec{p}|)$ take the form [7]:

$$
\begin{gathered}
S\left(\vec{p}, p_{4}\right)=\frac{Z(\vec{p})}{i \vec{p}+i \not p_{4} \alpha(\vec{p})+M(\vec{p})} \quad \omega_{F}(|\vec{p}|)=\frac{\alpha(|\vec{p}|)}{Z^{2}(|\vec{p}|)} \sqrt{\vec{p}^{2}+M^{2}(|\vec{p}|)} \\
M(|\vec{p}|)=\frac{m_{\chi}\left(m_{b}\right)}{1+b \frac{|\vec{p}|^{2}}{\Lambda^{2}} \log \left(e+\frac{|\vec{p}|^{2}}{\Lambda^{2}}\right)^{-\gamma}}+\frac{m_{r}\left(m_{b}\right)}{\log \left(e+\frac{|\vec{p}|^{2}}{\Lambda^{2}}\right)^{\gamma}},
\end{gathered}
$$

where $Z$ is the field renormalization function, $\alpha$ the energy renormalization function, $m_{b}$ the bare quark mass, $m_{\chi}\left(m_{b}\right)$ the chiral mass and $m_{r}\left(m_{b}\right)$ the renormalized running mass [7]. In the following we shall verify such behaviour and determine the relevant parameters.

\section{Results}

\subsection{Ghost form factor}

A careful analysis of the ghost form factor in the Hamiltonian limit $a_{t} \rightarrow 0$ shows that its UV behaviour agrees with Eq. (1.1), with $\gamma_{\mathrm{gh}}=1 / 2$, confirming continuum predictions, and $m=$ $0.21(1) \mathrm{GeV}$, see Fig. 1 (a). In the IR going to higher anisotropies increases the exponent $\kappa_{\mathrm{gh}}$, as shown in Fig. 1 (b), where we plot $|\vec{p}|^{\kappa_{m}} d(\vec{p})$, with $\kappa_{m}$ the IR exponent for $\xi=1$, as a function of the anisotropy. The limit $\xi \rightarrow \infty$ gives $\kappa_{\mathrm{gh}} \gtrsim 0.5$, confirming the GZ-scenario. This however disagrees with some continuum predictions $\kappa_{\mathrm{gh}}=1$, deriving from the assumption of the finiteness of the static ghost-gluon vertex. Whether this is indeed correct and algorithmic improvements could change the lattice result is still a matter of investigation. 


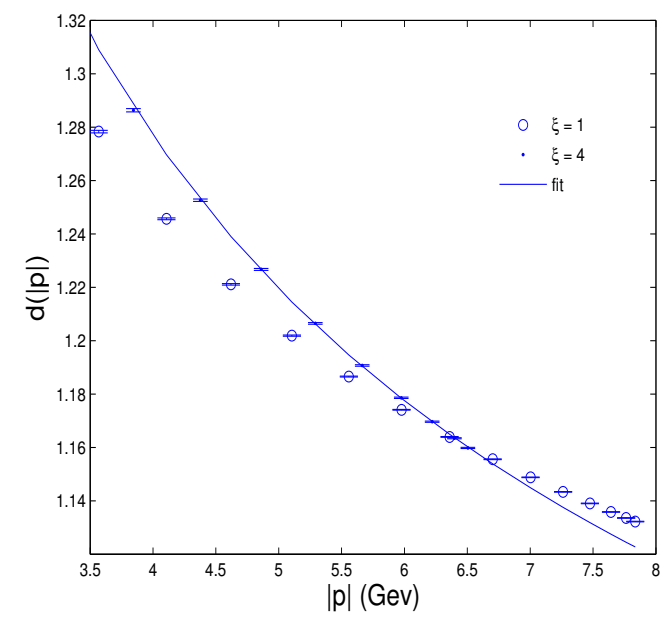

(a)



(b)

Figure 2: (a): UV behavior of $d(\vec{p})$ compared with Eq. (1.1). (b): IR behavior of $|\vec{p}|^{\kappa_{m}} d(\vec{p})$, both for different anisotropies $\xi$.

\subsection{Coulomb potential}

In Fig. 3 (a) we show $|\vec{p}|^{4} V_{C}(|\vec{p}|)$ as obtained from different anisotropies. Fitting the results to Eq. (1.1) we get, in the Hamiltonian limit $\xi \rightarrow \infty \sigma_{C}=2.2(2) \sigma$, as expected from Zwanziger's predictions [25].

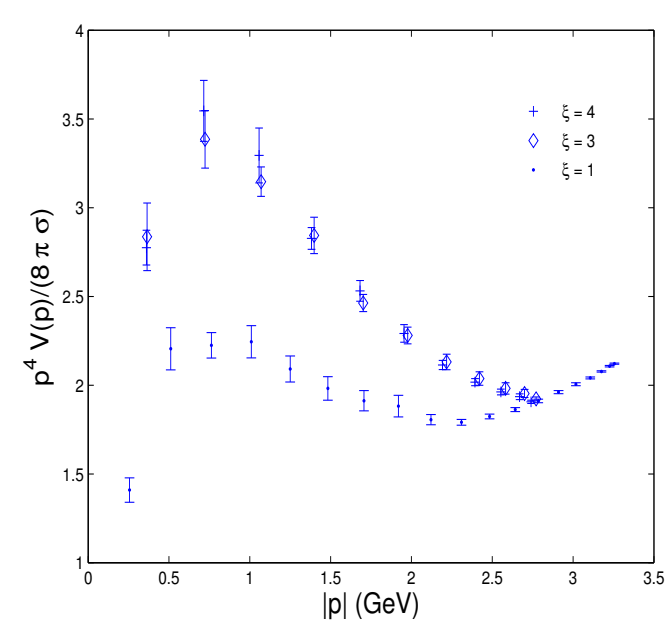

(a)



(b)

Figure 3: (a): Infrared behavior of $|\vec{p}|^{4} V_{C}(\vec{p}) /(8 \pi \sigma)$ for different anisotropies $\xi$. (b): Quark field renormalization function $Z(|\vec{p}|)$.

\subsection{Quark propagator}

Our calculations were all made on a set of configurations generated by the MILC collaboration 
[26], see [7] for details. The use of improved actions is crucial to establish the scaling properties of the Coulomb gauge quark propagators. This is very similar to the situation in Landau gauge, see e.g. $[27,28,29]$, whose techniques we have adapted to our case.

Fig. 3 (b) shows the scaling of the renormalization function $Z(|\vec{p}|)$ for configurations calculated at similar bare quark mass, while the RG-invariant functions $\alpha(|\vec{p}|)$ and $M(|\vec{p}|)$ are given in Fig. 4. Their behaviour agrees with theoretical expectations, see Eq. (1.2).

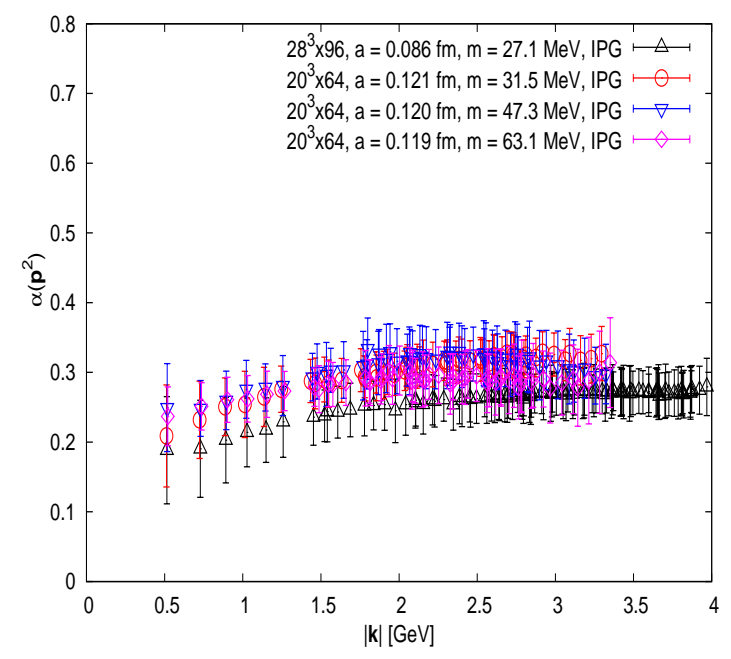

(a)

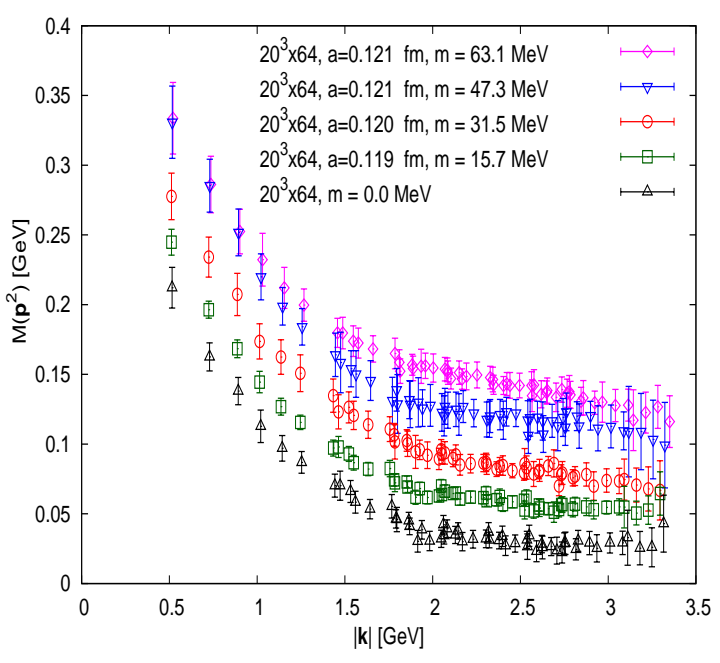

(b)

Figure 4: (a): Energy renormalization function $\alpha(|\vec{p}|)$. (b): Running mass $M(|\vec{p}|)$.

Our most interesting results are given in Fig. 5. Analogously to the gluon self-energy $\omega_{A}(|\vec{p}|)$, the quark self energy $\omega_{F}(|\vec{p}|)$ has a turn-over at $|\vec{p}| \sim 1 \mathrm{GeV}$, clearly departing from the behaviour of a free particle, and diverging in the IR, see Fig. 5 (a); although awaiting confirmation on larger lattices, this would in principle extend the Gribov argument for its disappearance from the physical spectrum to full QCD. Moreover, as Fig. 5 (b) shows, the running mass $M(|\vec{p}|)$ we obtain is quantitatively compatible with our phenomenological expectations from chiral symmetry breaking. Fitting it to Eq. (1.2) we obtain $b=2.9(1), \gamma=0.84(2), \Lambda=1.22(6) \mathrm{GeV}, m_{\chi}(0)=0.31(1) \mathrm{GeV}$, with $\chi^{2} /$ d.o.f. $=1.06$.

\section{Conclusions}

We have shown that the GZ confinement scenario is realized in Coulomb gauge. The ghost form factor $d(|\vec{p}|)$ is IR divergent with an exponent $\kappa_{\mathrm{gh}} \gtrsim 0.5$, which implies Gribov's no-pole condition and a dual-superconducting scenario [30]; the gluon propagator satisfies the Gribov formula, implying an IR diverging self-energy, and the Coulomb string tension is roughly twice the physical string tension. Moreover from the quark propagator we can easily extract the quark self energy $\omega_{F}(|\vec{p}|)$, which is also compatible with an IR divergent behaviour, and the running mass $M(|\vec{p}|)$, which gives a constituent quark mass of $m_{\chi}(0)=0.31(1) \mathrm{GeV}$.

This is in contrast to Landau gauge, where BRST symmetry seems to be non-perturbatively broken, violating the Kugo-Ojima confinement scenario [31], while the GZ confinement scenario 


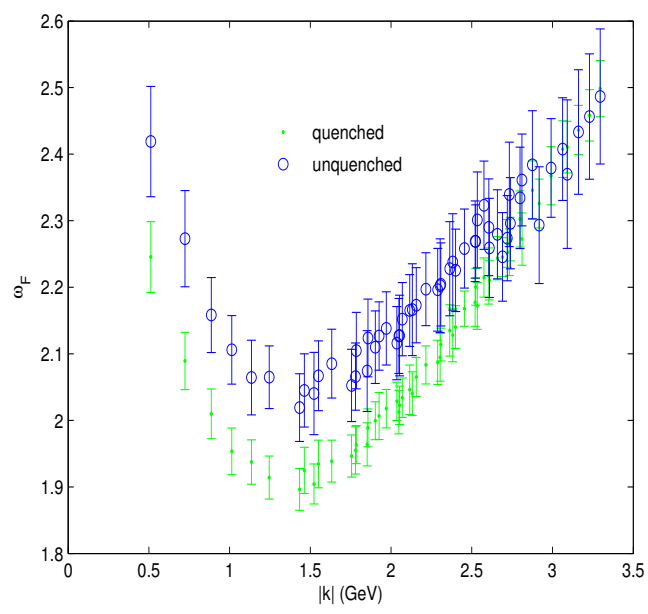

(a)

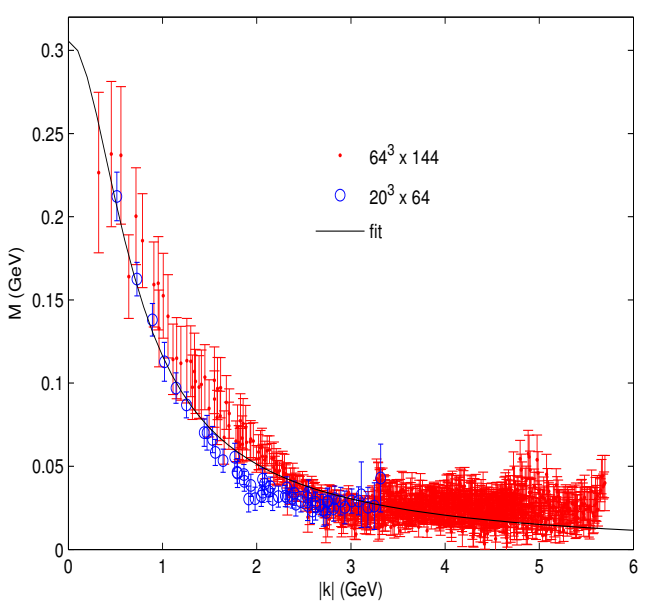

(b)

Figure 5: (a): Quark self energy $\omega_{F}(|\vec{p}|)$. (b): Running mass $M(|\vec{p}|)$ in the chiral limit $m_{b} \rightarrow 0$; see Eq. (1.2).

cannot be realized without the explicit introduction of an horizon function, see e.g. [32] for a recent review; its physical implications and how these can be related to the presence of dim- 2 condensates $[33,34,35]$ are an interesting issue still debated in the literature [36].

\section{References}

[1] V. N. Gribov, "Quantization of non-Abelian gauge theories," Nucl. Phys., vol. B139, p. 1, 1978.

[2] D. Zwanziger, "Lattice Coulomb Hamiltonian and static color-Coulomb field," Nucl. Phys., vol. B485, pp. 185-240, 1997.

[3] G. Burgio, M. Quandt, and H. Reinhardt, "Coulomb gauge gluon propagator and the Gribov formula," Phys. Rev. Lett., vol. 102, p. 032002, 2009.

[4] G. Burgio, M. Quandt, and H. Reinhardt, "BRST symmetry vs. Horizon condition in Yang-Mills theories," Phys. Rev., vol. D81, p. 074502, 2010.

[5] M. Quandt, H. Reinhardt, and G. Burgio, "The role of center vortices in Gribov's confinement scenario," Phys. Rev., vol. D81, p. 065016, 2010.

[6] H. Reinhardt, M. Quandt, and G. Burgio, "Temporal wilson loop in the hamiltonian approach in coulomb gauge," Phys. Rev. D, vol. 85, p. 025001, 2012.

[7] G. Burgio, M. Schrock, H. Reinhardt, and M. Quandt, "Running mass, effective energy and confinement: the lattice quark propagator in Coulomb gauge," Phys.Rev., vol. D86, p. 014506, 2012.

[8] G. Burgio, M. Quandt, and H. Reinhardt, "Ghost propagator and the Coulomb form factor from the lattice," Phys.Rev., vol. D86, p. 045029, 2012.

[9] A. P. Szczepaniak and E. S. Swanson, "Coulomb gauge QCD, confinement, and the constituent representation,” Phys. Rev., vol. D65, p. 025012, 2002.

[10] C. Feuchter and H. Reinhardt, "Variational solution of the Yang-Mills Schroedinger equation in Coulomb gauge," Phys. Rev., vol. D70, p. 105021, 2004. 
[11] D. Epple, H. Reinhardt, and W. Schleifenbaum, "Confining Solution of the Dyson-Schwinger Equations in Coulomb Gauge," Phys. Rev., vol. D75, p. 045011, 2007.

[12] G. Burgio, R. De Pietri, H. A. Morales-Tecotl, L. F. Urrutia, and J. D. Vergara, "The basis of the physical Hilbert space of lattice gauge theories," Nucl. Phys., vol. B566, pp. 547-561, 2000.

[13] A. Cucchieri and D. Zwanziger, "Fit to gluon propagator and Gribov formula," Phys. Lett., vol. B524, pp. 123-128, 2002.

[14] K. Langfeld and L. Moyaerts, "Propagators in Coulomb gauge from SU(2) lattice gauge theory," Phys. Rev., vol. D70, p. 074507, 2004.

[15] Y. Nakagawa, A. Nakamura, T. Saito, and H. Toki, "Infrared behavior of the Faddeev-Popov operator in Coulomb gauge QCD,” Phys.Rev., vol. D75, p. 014508, 2007.

[16] A. Voigt, E.-M. Ilgenfritz, M. Muller-Preussker, and A. Sternbeck, "The Effective Coulomb potential in SU(3) lattice Yang-Mills theory," Phys.Rev., vol. D78, p. 014501, 2008.

[17] Y. Nakagawa, A. Nakamura, T. Saito, and T. Toki, “The Coulomb gauge confinement scenario and the color-dependent quark potentials in lattice QCD simulations," Mod.Phys.Lett., vol. A23, pp. 2348-2351, 2008.

[18] Y. Nakagawa, A. Nakamura, T. Saito, and H. Toki, "Confining time-like gluon and confined spatial gluons in Coulomb gauge QCD,” Mod.Phys.Lett., vol. A23, pp. 2352-2355, 2008.

[19] G. Burgio, S. Caracciolo, and A. Pelissetto, "Algebraic algorithm for the computation of one loop Feynman diagrams in lattice QCD with Wilson fermions,” Nucl.Phys., vol. B478, pp. 687-722, 1996.

[20] G. Burgio, A. Feo, M. J. Peardon, and S. M. Ryan, "Gauge Theories on a 2+2 Anisotropic Lattice," Phys. Rev., vol. D67, p. 114502, 2003.

[21] K. Ishiguro, T. Suzuki, and T. Yazawa, "Effective monopole action at finite temperature in SU(2) gluodynamics," JHEP, vol. 0201, p. 038, 2002.

[22] I. L. Bogolubsky et al., "Landau gauge ghost and gluon propagators in SU(2) lattice gauge theory: Gribov ambiguity revisited,” Phys. Rev., vol. D74, p. 034503, 2006.

[23] I. L. Bogolubsky et al., "Improved Landau gauge fixing and the suppression of finite-volume effects of the lattice gluon propagator," Phys. Rev., vol. D77, p. 014504, 2008.

[24] Y. Nakagawa, A. Nakamura, T. Saito, and H. Toki, "Scaling study of the gluon propagator in Coulomb gauge QCD on isotropic and anisotropic lattices," Phys.Rev., vol. D83, p. 114503, 2011.

[25] D. Zwanziger, "No confinement without Coulomb confinement," Phys.Rev.Lett., vol. 90, p. 102001, 2003.

[26] A. Bazavov, D. Toussaint, C. Bernard, J. Laiho, C. DeTar, et al., "Nonperturbative QCD simulations with 2+1 flavors of improved staggered quarks," Rev.Mod.Phys., vol. 82, pp. 1349-1417, 2010.

[27] P. O. Bowman, U. M. Heller, and A. G. Williams, "Lattice quark propagator with staggered quarks in Landau and Laplacian gauges,” Phys.Rev., vol. D66, p. 014505, 2002.

[28] P. O. Bowman, U. M. Heller, D. B. Leinweber, M. B. Parappilly, A. G. Williams, et al., "Unquenched quark propagator in Landau gauge," Phys.Rev., vol. D71, p. 054507, 2005.

[29] M. B. Parappilly et al., "Scaling behavior of quark propagator in full QCD," Phys.Rev., vol. D73, p. $054504,2006$. 
[30] H. Reinhardt, "The dielectric function of the QCD vacuum," Phys. Rev. Lett., vol. 101, p. 061602, 2008.

[31] T. Kugo and I. Ojima, "Local Covariant Operator Formalism of Nonabelian Gauge Theories and Quark Confinement Problem,” Prog. Theor. Phys. Suppl., vol. 66, p. 1, 1979.

[32] N. Vandersickel and D. Zwanziger, “The Gribov problem and QCD dynamics," Phys.Rept., vol. 520, pp. 175-251, 2012.

[33] G. Burgio, F. Di Renzo, G. Marchesini, and E. Onofri, " $\Lambda^{2}$-contribution to the condensate in lattice gauge theory," Phys. Lett., vol. B422, pp. 219-226, 1998.

[34] R. Akhoury and V. I. Zakharov, "On non perturbative corrections to the potential for heavy quarks," Phys. Lett., vol. B438, pp. 165-172, 1998.

[35] P. Boucaud et al., "Lattice calculation of $\frac{1}{p^{2}}$ corrections to $\alpha(s)$ and of $\Lambda_{Q C D}$ in the $\widetilde{M O M}$ scheme," JHEP, vol. 04, p. 006, 2000.

[36] A. Cucchieri, D. Dudal, T. Mendes, and N. Vandersickel, "Modeling the Gluon Propagator in Landau Gauge: Lattice Estimates of Pole Masses and Dimension-Two Condensates," Phys.Rev., vol. D85, p. 094513, 2012. 